\title{
Manner of Execution and Efficacy of Reception in Men's Beach Volleyball
}

\author{
Jose M. Palao ${ }^{1}$, Ana Lopez-Martinez ${ }^{2}$, David Valades ${ }^{3}$, Elena Hernandez ${ }^{4}$
}

\begin{abstract}
Affiliations: 'University of Wisconsin-Parkside, Kenosha, United States, ${ }^{2}$ Catholic University of San Antonio, Murcia, Spain, ${ }^{3}$ University of Alcala, Alcala de Henares, Spain, ${ }^{4}$ Pablo de Olavide University, Sevilla, Spain
\end{abstract}

Correspondence: J.M. Palao, University of Wisconsin-Parkside, Department of Health, Exercise Science and Sport Management, SAC D140A, Kenosha, WI 53144, United States, E-mail: palaojm@gmail.com

ABSTRACT The purpose of this study was to determine the effect of the way the reception was executed on the efficacy of the reception in men's beach volleyball. The sample of this study was composed of 5,161 receptions, corresponding to 84 matches (179 sets) of the Men's Beach Volleyball World Tour organized by the Fédération Internationale de Volleyball (FIVB). The sample included only confrontations between the first 30 teams of the World Tour (FIVB ranking). The variables studied were: a) manner of serve execution: standing, power jump serve, and floating jump serve; b) reception technique (bump, overhead, and other); c) zone of reception: the court was divided into 10 equal zones; d) reception efficacy; e) spike efficacy; and f) rally result: win, continuity, or loss. The bump reception involved the highest efficacy coefficient, the number of receptions that allowed all options, and it was the most used reception. The power jump serve was the type of serve that most limited the receivers. This limitation was found when the serve required the receivers to move. The same tendency was found in the reception of the floating jump serve and standing serve, although not in the interference zone. Reception efficacy has a direct relationship with spike efficacy and winning the rally. The reception is the foundation for building a team's attack. The data found in the present study can be used as a reference to guide match analysis and practices in men's performance-level beach volleyball.

KEY WORDS sport, match analysis, scouting, observation

$@ M J S S M o n t e n e g r o$

RECEPTION IN MEN'S BEACH VOLLEYBALL

http://mjssm.me/?sekcija=article\&artid=178

\section{Introduction}

Athletes, teams, and coaches work to improve performance in competition. However, not all aspects of the sport have the same importance. It is possible to find many studies in sport science about performance indicators of different sports. The technical-tactical performance indicators can be established in relation to the way that points are scored or in relation to how the score is achieved (Hughes \& Barlett, 2002). In beach volleyball, the skills that are executed are discrete, but their executions in the game are repeated cyclically (neutralizing the opponent's actions, preparing the team's attack, and attacking). The skills related to the way a team is organized and attempts to score are called actions of continuity (reception, dig, and set), and the skills related to the possibility of scoring are called terminal actions (serve, attack, and block).

More studies and information are available in relation to terminal actions than to actions of continuity (Medeiros, Palao, Marcelino, \& Mesquita, 2014; Mesquita, Palao, Marcelino, \& Afonso, 2013). Additionally, beach volleyball is a relatively new sport. Its first World Championship was in 1987, and its first inclusion in the Olympic Games was in 1996. Therefore, the same volume of studies as in other sports does not exist. Most of the information about the reception and the dig can be deduced by studies about the serve and the attack (Buscà et al., 2012; Jimenez-Olmedo, Penichet-Tomas, Saiz-Colomina, Martínez-Carbonell, and Jove-Tossi, 2012; López-Martínez \& Palao, 2009), respectively, because these actions are attempts to neutralize the serve and attack actions to organize the team's offence. The information about reception in indoor volleyball is not applicable to beach volleyball due to the differences between these sports (e.g., number of players, surface area to be covered by players, type of surface, specialization 
of the players, target zone, etc.). In beach volleyball, the efficacy of the reception is high. In nine out of ten receptions, the execution allows the team to build an attack (Lacerda \& Mesquita, 2003; Lopez-Martinez \& Palao, 2009). The serve only reduces the reception efficacy when the serve moves the receivers (Homberg \& Papageorgiou, 1995). The deep right zone (Lacerda \& Mesquita, 2003) and the zone between the receivers (Lacerda \& Mesquita, 2003; Lopez-Martinez \& Palao, 2009) are the zones where most of the errors are found. The reason for this is probably the difficulty of moving in the sand, in addition to communication problems in the zone between the receivers (Noël, Hüttermann, van der Kamp, \& Memmert, 2016; Smith, 2006). Most of the information available about reception is obtained from serve studies.

The fact that there is little available information about the way the reception is executed may mean that coaches are using subjective data to guide and analyse their training and competition processes. Also, this deficit of information makes it difficult to know the effect of rule changes and the sport's evolution. Previous researchers have focused on the terminal actions; however, the analysis of these aspects alone does not give an accurate perspective of the game. The actions of continuity do not allow teams to get points, but they are the ones that allow the terminal actions to do it. The purpose of this study was to determine the effect of the way the reception was executed on the efficacy of the reception in men's beach volleyball.

\section{Methods}

The sample of this study was composed of 5,161 receptions carried out by 91 players (from 23 countries, with an average height of $1.93 \pm 0.06$ metres, an average weight of $88.7 \pm 6.3$ kilograms, and an average age of $30.3 \pm 5.3$ years), corresponding to 84 matches (179 sets) of the 2008 Men's Beach Volleyball World Tour organized by the Fédération Internationale de Volleyball (FIVB). The sample only included confrontations between the first 30 teams of the World Tour (FIVB ranking). Sample selection was made according to the following criteria: a) three levels of teams were established (those that were classified 1 st-10th, 11th-20th, and 21st-30th). An equal number of matches of the six possible combinations of confrontations between these three levels was included, and b) a maximum of four matches or twelve sets per team was included in the sample. The video recordings of the matches were obtained from the researchers' records and from recordings by the Spanish National Coach.

The study's design was descriptive, correlational, and transversal. The observation instrument that was used was a category system (Anguera, 2003). The variables that were studied included: a) manner of serve execution: standing, power jump serve, and floating jump serve (Palao, Manzanares, \& Ortega, 2015); b) reception technique (bump, overhead, and other); c) zone of reception (Figure 1): the court was divided into 10 zones; d) reception efficacy (Coleman, Neville, \& Gordon, 1969); e) spike efficacy (Coleman et al., 1969); and f) rally result: win, continuity, or loss. The studied variables are part of the observational instrument develop and validated by Palao and Manzanares (2009), and Palao et al. (2015).

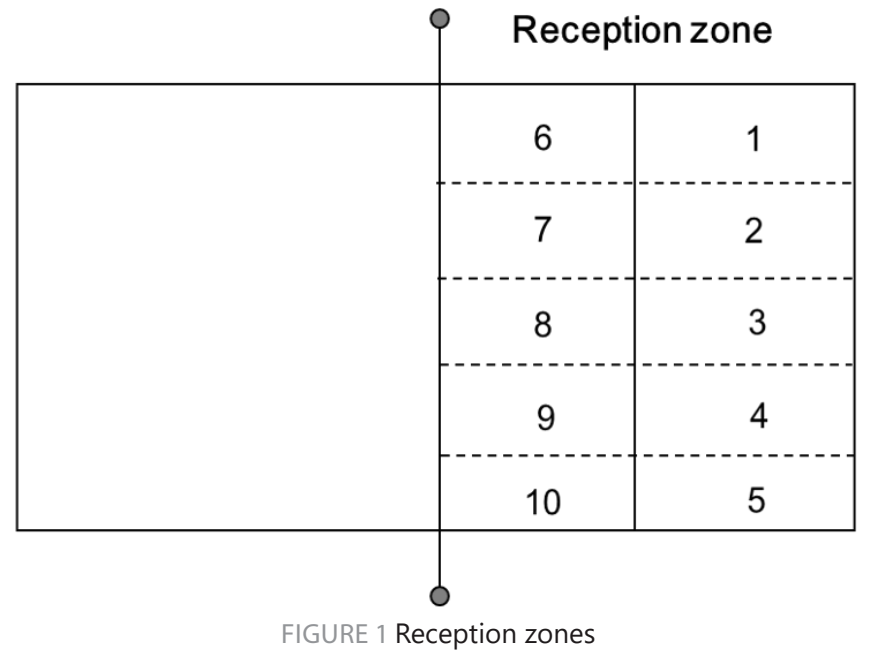

The zone of serve destination was determined by the player position in reception (zones 2 and 4), and the court zones were established in relation to player movement. It was considered a displacement of the players to another zone when the players' second foot lost contact with the sand when taking a step. Reception and spike efficacy performance were evaluated in relation to their success and the options they gave for the following actions (Coleman et al., 1969). The four levels to categorize reception performance were: a) Error: point for the opponent (0); b) no attack options (1); c) limited attack options (2); and d) maximum attack options (3). The five levels distinguished to categorize spike performance were: a) error/point for the opponent (0); b) maximum opponent attack options (1); c) limited attack options for the opponent (2); d) no opponent attack options (3); and e) point (4). With the categories of serve performance, an efficacy coefficient (sum of the attempts per category multiplied by the value of the category and divided by the total attempts (Coleman et al., 1969)) and the point-to-error ratio were calculated. 
The matches were analysed through systematic observation by four experienced observers, who were trained using the methodology described by Anguera (2003). The matches were recorded from a posterior view by researchers or coaches. The recording included the entire volleyball court. The coding was done in a spreadsheet. The quality of the data registered by the observers was monitored through the elimination of incongruities, random review of the analysis, and spreadsheet cell blocking and/or protecting. Ten per cent of the sample was re-analysed to ensure the quality of the data. After training and during the analysis, the inter-observer and intra-observer reliability percentages of the studied variables were calculated between the observer and one of the researchers (Anguera, 2003). The inter- and intra-reliability of two separate observations was calculated to guarantee sufficient quality of the observation system. An inter-reliability index of 0.87 and intra-reliability index of 0.98 were found (intra-class correlation coefficient and Kappa index).

Actions in which the video recording did not allow all variables to be properly observed were considered lost values, and they were not taken into account in the data analysis. A descriptive analysis of the different variables was carried out. Contingency tables and non-parametric $\chi^{2}$ (Chi-square) tests were used to establish the relationships between the nominal variables. The Student $\mathrm{t}$-test for independent samples was used to assess differences between the manner of execution of the reception and reception efficacy and rally result. Statistical significance was set at 0.05 .

\section{Results}

Significant differences between technique and efficacy ( $p<.001)$ were found (Table 1$)$. The bump was the technique that was most used $(94.7 \%)$. The use of the bump involved a significantly higher number of receptions that allowed the maximum options (56.7\%). The overhead and other receptions involved a significantly higher number of errors (16.7\% and $20.8 \%)$ and receptions that limited options $(66.7 \%$ and $54.7 \%)$.

TABLE 1 Technique used in reception and efficacy in men's beach volleyball

\begin{tabular}{|c|c|c|c|c|c|c|c|c|}
\hline & \multicolumn{2}{|c|}{ Bump } & \multicolumn{2}{|c|}{ Overhead } & \multicolumn{2}{|c|}{ Other } & \multicolumn{2}{|c|}{ Total } \\
\hline & $\mathbf{n}$ & $\%$ & $\mathbf{n}$ & $\%$ & $\mathbf{n}$ & $\%$ & n & $\%$ \\
\hline Error & 73 & 1.5 & $1^{*}$ & 16.7 & $55^{*}$ & 20.8 & 129 & 2.5 \\
\hline No options & 72 & 1.5 & 0 & 0.0 & 24 & 9.1 & 96 & 1.9 \\
\hline Options & 1934 & 40.3 & $4^{*}$ & 66.7 & $145^{*}$ & 54.7 & 2083 & 41.1 \\
\hline Max options & $2719^{*}$ & 56.7 & 1 & 16.7 & 41 & 15.5 & 2761 & 54.5 \\
\hline Occurrence & 4798 & 94.7 & 6 & 0.1 & 265 & 5.2 & 5069 & 100.0 \\
\hline Coefficient & \multicolumn{2}{|c|}{2.52} & \multicolumn{2}{|c|}{1.83} & \multicolumn{2}{|c|}{1.65} & \multicolumn{2}{|c|}{2.47} \\
\hline Ratio & \multicolumn{2}{|c|}{$1: 0.03$} & \multicolumn{2}{|c|}{$1: 1.00$} & \multicolumn{2}{|c|}{$1: 1.34$} & \multicolumn{2}{|c|}{$1: 0.05$} \\
\hline
\end{tabular}

Significant differences between serve type and reception efficacy $(\mathrm{p}<.001)$ were found (Table 2$)$. Reception of the power jump serve (42.7\%), and reception of the floating jump serve $(37.7 \%)$ occurred the most often. The reception of the standing serve and the floating jump serve involved a significantly higher number of serves that allowed the maximum options (64.8\% and 59.3\%, respectively). The power jump serve involved a significantly higher number of reception errors (8.9\%) and receptions that did not allow the team to attack $(2.5 \%)$.

\begin{tabular}{|c|c|c|c|c|c|c|c|c|}
\hline & \multicolumn{2}{|c|}{ Standing serve } & \multicolumn{2}{|c|}{ Power jump serve } & \multicolumn{2}{|c|}{ Floating jump serve } & \multicolumn{2}{|c|}{ Total } \\
\hline & $\mathbf{n}$ & $\%$ & $\mathbf{n}$ & $\%$ & $\mathbf{n}$ & $\%$ & $\mathbf{n}$ & $\%$ \\
\hline Error & 15 & 1.5 & $195 *$ & 8.9 & 36 & 1.9 & 246 & 4.8 \\
\hline No options & 10 & 1.0 & $56 *$ & 2.5 & 28 & 1.4 & 94 & 1.8 \\
\hline Options & 328 & 32.7 & 1013 & 46.4 & 721 & 37.9 & 2062 & 40.3 \\
\hline Max options & $650 *$ & 64.8 & $920 *$ & 42.1 & 1144 & 59.3 & 2714 & 53.0 \\
\hline Occurrence & 1003 & 19.6 & 2184 & 42.7 & 1929 & 37.7 & 5116 & 100.0 \\
\hline Coefficient & \multicolumn{2}{|c|}{2.61} & \multicolumn{2}{|c|}{2.22} & \multicolumn{2}{|c|}{2.54} & \multicolumn{2}{|c|}{2.42} \\
\hline Ratio & \multicolumn{2}{|c|}{$1: 0.02$} & \multicolumn{2}{|c|}{$1: 1.21$} & \multicolumn{2}{|c|}{$1: 1.03$} & \multicolumn{2}{|c|}{$1: 0.09$} \\
\hline
\end{tabular}

Note. ${ }^{*} \mathrm{P}<0.001$.

Significant differences were found in the reception of the standing serve in the different zones of reception $(\mathrm{p}<.001)$ (Figure 2). The serves to zones 2 and 4 had a significantly higher number of receptions that allowed the opponent team all attack options. The deep and lateral receptions (zones 1 and 5) obtained significantly lower efficacies. Significant differences were found in the reception of the power jump serve in the different zones of reception $(\mathrm{p}<.001)$. The serves directed to zones 2 and 4 had a significantly higher number of receptions that allowed the opponent team all attack options. The receptions carried out in zones 1, 3, 5, 6, 7, 8, 9, and 10 ob- 
tained significantly lower efficacies than receptions carried out in zones 2 and 4 . Significant differences were found in the reception of the floating jump serve in the different zones of reception $(\mathrm{p}<.001)$. The receptions carried out in zones 2 and 4 had a significantly higher number of receptions that allowed the team all attack options. The deep and lateral receptions (zones 1 and 5) obtained significantly lower efficacies. Regardless of the type of serve, the reception done in the short zones (close to the net) occurred infrequently.
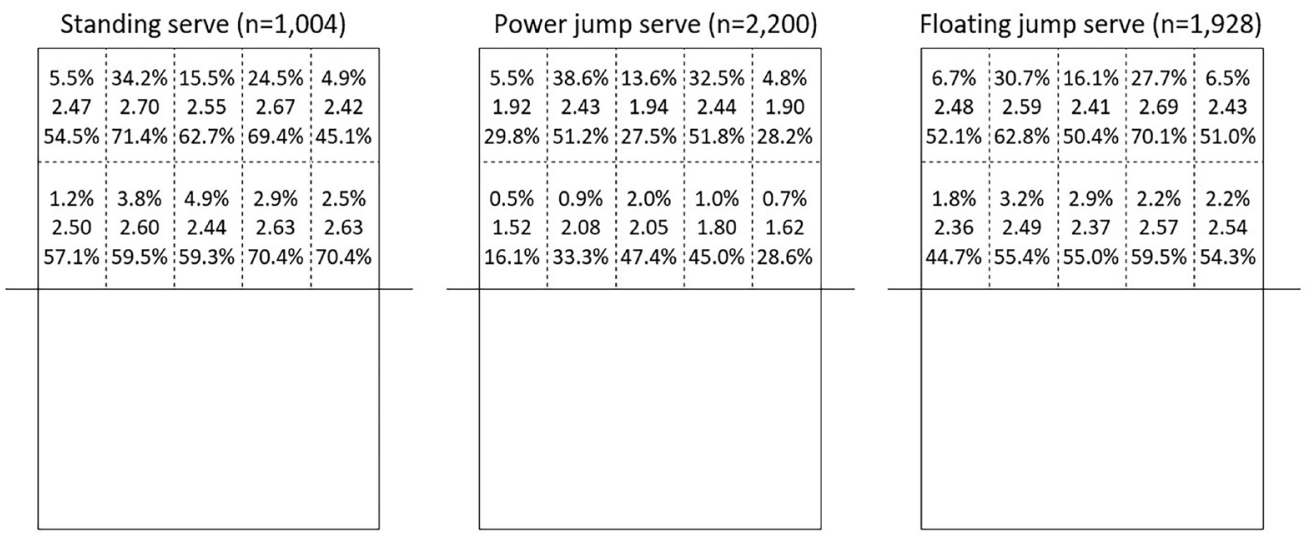

FIGURE 2 Occurrence (percentage), efficacy coefficient of the reception, and percentage of receptions that allowed maximum attack options in relation to different types of serve and the reception zone in men's beach volleyball

Significant differences were found between reception efficacy and spike ( $\mathrm{p}<.001)$ (Table 3$)$. The reception that allowed the spiker all attack options had a significantly higher number of points and lower number of errors. The spike efficacy was higher when the reception allowed maximal attack options.

\begin{tabular}{|c|c|c|c|c|c|c|}
\hline \multirow[b]{3}{*}{ Spike efficacy } & \multicolumn{4}{|c|}{ Reception efficacy } & & \\
\hline & \multicolumn{2}{|c|}{ Limited attack options } & \multicolumn{2}{|c|}{ Max attack options } & \multicolumn{2}{|c|}{ Total } \\
\hline & $\mathbf{n}$ & $\%$ & $\mathbf{n}$ & $\%$ & $\mathrm{n}$ & $\%$ \\
\hline Error & 337 & 16.6 & $350 *$ & 12.8 & 687 & 14.4 \\
\hline Max opp. options & 70 & 3.4 & 73 & 2.7 & 143 & 3.0 \\
\hline Limited opp. attack options & 458 & 22.5 & 533 & 19.5 & 991 & 20.8 \\
\hline No opponent attack & 148 & 7.3 & 167 & 6.1 & 315 & 6.6 \\
\hline Point & 1023 & 50.2 & $1610 *$ & 58.9 & 2633 & 55.2 \\
\hline Occurrence & 2036 & 42.7 & 2733 & 57.3 & 4769 & 100.0 \\
\hline Coefficient & \multicolumn{2}{|c|}{2.71} & \multicolumn{2}{|c|}{2.96} & \multicolumn{2}{|c|}{2.85} \\
\hline Ratio & \multicolumn{2}{|c|}{$1: 2.28$} & \multicolumn{2}{|c|}{$1: 2.10$} & \multicolumn{2}{|c|}{$1: 2.18$} \\
\hline Efficacy & \multicolumn{2}{|c|}{$33.7 \%$} & \multicolumn{2}{|c|}{$46.1 \%$} & \multicolumn{2}{|c|}{$40.8 \%$} \\
\hline
\end{tabular}

Note. ${ }^{*} \mathrm{P}<0.001$.

Significant differences were found between reception efficacy and rally result $(\mathrm{p}<.001)$ (Table 4$)$. The reception that allowed maximal attack options had significantly higher possibilities of winning the rally. The reception that limited the attack options had significantly lower possibilities of winning the rally.

TABLE 4 Relationship between reception efficacy and side-out result in men's beach volleyball

\begin{tabular}{lccccccccc} 
& \multicolumn{2}{c}{ Loss } & \multicolumn{2}{c}{ Continuity } & \multicolumn{2}{c}{ Win } & \multicolumn{2}{c}{ Total } \\
\hline & $\mathbf{n}$ & $\%$ & $\mathbf{n}$ & $\%$ & $\mathbf{n}$ & $\%$ & $\mathbf{n}$ & $\%$ \\
\hline Error & 245 & 26.3 & 0 & 0.0 & 0 & 0.0 & 245 & 4.7 \\
No options & 10 & 1.1 & 73 & 4.7 & 13 & 0.5 & 96 & 1.9 \\
Limit options & 334 & 35.8 & 699 & 45.3 & $1051 *$ & 38.8 & 2084 & 40.2 \\
Max options & 343 & 36.8 & 772 & 50.0 & $1646^{*}$ & 60.7 & 2761 & 53.2 \\
\hline Occurrence & 932 & 18.0 & 1544 & 29.8 & 2710 & 52.3 & 5186 & 100 \\
\hline
\end{tabular}

Note. ${ }^{*} \mathrm{P}<0.001$

\section{Discussion}

The purpose of this paper was to assess the effect of the manner of reception execution on the reception's efficacy in men's beach volleyball. The values found regarding efficacy demonstrate why the bump is the type of reception that is most used. In part, these results are logical given the goal of this technique and beach volleyball rules, which establish that ball contacts must be clean. The bump allows players to reduce 
the speed of the ball, to intercept the ball at a higher range of angles, and to adapt the different trajectories. The occurrence found in the rest of the actions is very low $(<5 \%)$, and those actions have low efficacy. These techniques are used as a resource when the receiver does not get his body behind the ball. The use of these techniques has decreased with the reduction in court size $(8 \times 8 \mathrm{~m})$.

With regard to the type of serve, the reception of the power serve presented lower efficacy than the floating jump serve and the standing serve did. The reason for this result is probably because the speed of the ball does not allow the player to execute the reception properly. This aspect is confirmed with the information about the efficacy of the reception in the different zones. In the reception of the power jump serve, if the players have to move (laterally) or the ball goes to the interference zone between the players, the efficacy of the reception is significantly lower. The same tendency is observed in the rest of the serves although the reduction of the efficacy is also lower. The fact that there is less uncertainty with the floating and jump serves reduces the problems in reception in the interference zone between receivers (zone 3). The values found show that the analysis of the reception should take into consideration the type of serve. This aspect must be considered in match analysis and in practice. For example, although the general values are similar, the difference is the quality of the reception. The power jump serve only allows maximal attack options in $30 \%$ of receptions.

The relationship between reception efficacy, spike efficacy, and the possibility of winning the rally confirms the importance of the reception in the game. This relationship, called a "second order relationship" because it is the second-to-last action before the attack, has been confirmed in indoor volleyball (Eom \& Schultz, 1992; Palao, Santos, / Ureña, 2006).

Data found can be used for planning and monitoring practice and competition for players of a similar level. The data can be considered as normative profiles for the peak performance level and can be used as a reference to be achieved by players in formation. The results show that reception analysis should take into consideration the type of serve and, if possible, the serve speed (Buscà, Moras, Peña, AND Rodríguez-Jiménez, 2012; Palao \& Valades, 2014). The use of the observational methodology to collect the data increases the risk of error in the measurement of court zone usage (Koch et al., 2009; Mauthner, Koch, Tilp, \& Bischof, 2007). However, the goals of the study were to give applicable and useful data to coaches and players. The authors believe that the criteria followed to determine the serve destination according to players' displacement allow coaches and players to easily interpret and use the data.

The bump reception involved a higher efficacy coefficient (i.e., a higher number of receptions that allowed all options), and it was the most used reception. The power jump serve was the type of serve that most limited the receptors. This limitation was found when the serve moved the receivers. The same tendency was found in the reception of the floating jump serve and standing serve, although not in the interference zone. Reception efficacy has a direct relationship with spike efficacy and winning the rally. The reception is the foundation for building the team's attack. The data found in the present study can be used as a reference to guide match analysis and practices in men's performance-level beach volleyball. Future studies should attempt to assess whether there is any relationship between the receiver's actions and their posterior actions (e.g., type of attack, number of receptions done, etc.).

\section{Acknowledgements}

This research has been possible thanks to the research grant "Technical and tactical performance indicators and goal establishment in volleyball and beach volleyball" (2008-2010) supported by the Seneca Foundation (Región de Murcia, Spain).

\section{REFERENCES}

Anguera, M. T. (2003). Observational methods (general). In R. Fernández-Ballesteros (Ed.), Encyclopedia of Psychological Assessment, Vol. 2 (pp. 632-637). London: Sage.

Buscà, B., Moras, G., Peña, J., \& Rodríguez-Jiménez, S. (2012). The influence of serve characteristics on performance in men's and women's high-standard beach volleyball. Journal of Sports Sciences, 30(3), 269-276. doi: 10.1080/02640414.2011.635309

Coleman, J. E., Neville, B., \& Gordon, B. (1969). A statistical system for volleyball and its use in Chicago Women's Association. International Volleyball Review, 17, 72-73.

Eom, H.J., \& Schutz, R.W. (1992). Statistical analyses of volleyball team performance. Research Quarterly for Exercise and Sport, 63(1), 11-18.

Homberg, S., \& Papageorgiu, A. (1995). Handbook for beach volleyball. Aachen: Meyer \& Meyer Sport.

Hughes, M. D., \& Bartlett, R. M. (2002). The use of performance indicators in performance analysis. Journal of sports sciences, 20(10), 739-754.

Jimenez-Olmedo, J. M., Penichet-Tomas, A., Saiz-Colomina, S., Martínez-Carbonell, J. A., \& Jove-Tossi, M. A. (2012). Serve analysis of professional players in beach volleyball. Journal of Human Sport \& Exercise, 7(3), 706-713.

Koch, C., Mauthner, T., Tilp, M., \& Schrapf, N. (2009) Evaluation of visual position estimation in beach volleyball. International Journal of Performance Analysis in Sport, 9(3), 332-43. 
Lacerda, D., \& Mesquita, I. (2003). Analysis of the offensive process on the side out in elite beach volleyball. EF deportes, 61, 9.

López-Martinez, A. B. \& Palao, J. M. (2009). Effect of serve execution on serve efficacy in men's and women's beach volleyball. International Journal of Applied Sports Sciences, 21(1), 1-16.

Mauthner, T., Koch, C., Tilp, M., \& Bischof, H. (2007) Visual tracking of athletes in beach volleyball using a single camera. Journal of Computer Science in Sports, 6(2), 21-35.

Medeiros, A. I. A., Palao, J. M., Marcelino, R., \& Mesquita, I. (2014). Systematic review on sports performance in beach volleyball from match analysis. Revista Brasileira de Cineantropometria \& Desempenho Humano, 16(6), 698-708.

Mesquita, I., Palao, J. M., Marcelino, R. \& Afonso, J. (2013). Chapter 29. Indoor volleyball and beach volleyball (pp. 367-379). In T. McGarry, P. O’Donoghue, \& J. Sampaio (editors). Routledge Handbook of Sports Performance Analysis. London: Routledge.

Noël, B., Hüttermann, S., van der Kamp, J., \& Memmert, D. (2016). Courting on the beach: how team position implicitly influences decision-making in beach volleyball serves. Journal of Cognitive Psychology, 28(7), 868-876.

Palao, J. M., \& Valades, D. (2014). Serve speed peaks in FIVB World Tour beach volleyball (2005-2012). Acta Kinesiologica, 8(2), 7-10.

Palao, J. M., Manzanares López, P., \& Ortega, E. (2015). Design and validation of an observational instrument for technical and tactical actions in beach volleyball. Motriz: Revista de Educação Física, 21(2), 137-147.

Palao, J. M., Santos, J. A., \& Ureña, A. (2006). Effect of reception and dig efficacy on spike performance and manner of execution in volleyball. Journal of Human Movement Studies, 51(4), 221-238.

Smith, R. (2006). Movement in the sand: Training implications for beach volleyball. Strength \& Conditioning Journal, 28(5), 19-21. 